\title{
Insight into the Attitudes and Experiences of Adults with Non-Communicable Diseases a Year into the COVID-19 Pandemic: The Malta Experience
}

\author{
Sarah Cuschieri ${ }^{a}$ Stephan Grech ${ }^{b}$ Victor Grech ${ }^{c}$ \\ ${ }^{a}$ Anatomy Department, Faculty of Medicine and Surgery, University of Malta, Msida, Malta; ${ }^{\mathrm{b} T r a u m a}$ and \\ Orthopaedic Department, Mater Dei Hospital, Msida, Malta; 'Paediatric Department, Mater Dei Hospital, \\ Msida, Malta
}

\section{Keywords}

Non-communicable diseases · Coronavirus · Delivery of health care $\cdot$ Syndemic $\cdot$ Malta

\begin{abstract}
Introduction: Non-communicable diseases (NCDs) have been a global epidemic long before the advent of COVID-19. Understandably, with the onset of COVID-19, health priorities shifted. The aim of this study was to explore the impact of COVID-19 through attitudes and experiences on the health and well-being of the Malta adult population suffering from NCDs, a year into COVID-19. Methods: An anonymous survey was distributed online between February 1 and 26, 2021 using Google Forms ${ }^{\circ}$. This assessed the impact of COVID-19 on medical care, intention to take a COVID-19 vaccine, and whether COVID-19 was acquired. Quantitative and qualitative analyses were performed. Results: Out of the 1,034 participants, $34.82 \%(95 \% \mathrm{Cl}, 31.97-37.77 ; n=360)$ reported to suffer from NCDs (single NCD, $n=276 ; 2$ NCDs, $n=$ $56 ; 3 \mathrm{NCDs}, n=28)$ with $6.94 \%(95 \% \mathrm{Cl}, 4.71-10.09)$ of these reported acquiring COVID-19. Since COVID-19, the NCD population visited the general practitioners less $(47 \%)$ than those without NCDs (32\%) ( $p \leq 0.001)$. With a consensus of concern and fear, "I rather skip check-ups than risk getting
\end{abstract}

COVID-19 in waiting room or clinic." Postponement and cancellations of medical appointments were reported: "had to do blood tests privately as health centre was not doing them" and "delayed treatment and still awaiting further appointments that were postponed more than once." The majority intended to take COVID-19 vaccine. Conclusions: It is clear that individuals with NCDs have experienced a general negative effect on their medical care. It's recommended that a dual action strategy is embraced to ensure that both NCDs and COVID-19 are treated simultaneously, leaving no one behind.

(c) 2021 The Author(s).

Published by S. Karger AG, Basel

\section{Introduction}

Non-communicable diseases (NCDs) have been a public health concern for decades and constitute a substantial global burden [1]. NCDs are a collection of chronic diseases, such as cardiovascular disease, stroke, cancer, diabetes, and mental health illness, that are responsible for high population morbidity and mortality. The year 2020 saw the emergence of novel coronavirus severe acute respiratory syndrome coronavirus-2 (SARS-CoV2, with the disease referred as COVID-19) that brought the world karger@karger.com www.karger.com/dmj

Karger $\stackrel{\text { ' }}{5}$

GOPEN ACCESS
(C) 2021 The Author(s)

Published by S. Karger AG, Basel

This is an Open Access article licensed under the Creative Commons Attribution-NonCommercial-4.0 International License (CC BY-NC) (http://www.karger.com/Services/OpenAccessLicense), applicable to the online version of the article only. Usage and distribution for commercial purposes requires written permission.
Correspondence to:

Sarah Cuschieri, sarah.cuschieri@um.edu.mt 
to a standstill, with a number of healthcare systems collapsing $[2,3]$. The mode of transmission of COVID-19 is through direct contact or indirect contact with infected individuals through infectious respiratory droplets, secretions, or saliva. Respiratory droplets transmission can occur as far as $1 \mathrm{~m}$, while indirect infectivity can occur by having contact with a contaminated surface or object/s. Practicing regular hand hygine and wearing masks are protective ways to avoid contracting the virus [4].

SARS-CoV2 has been described to have an envelope with spike glycoprotein that enables the virus to enter human cells (through angiotensin-converting enzyme 2 ACE2). This is also responsible for host-to-host transmission [5]. The ACE2 can be found in a number of different epithelium although ACE2 is mostly found in the human respiratory system. The activation of ACE 2 increases proinflammatory responses [6]. Hence, individuals suffering from severe COVID-19 might have an imbalance in the ACE2 activation pathways, with an increased pro-inflammatory response [7].

Governments and public health authorities around the world instituted containment measures to slow down viral spread, drastically shifting health priorities. These measures have been anticipated to result in a multifaceted adverse effects upon the NCD population, through poor medical care and negative behavioural outcomes [8].

The European country of Malta (457,267 population) situated in the middle of the Mediterranean Sea reported the first COVID-19 case on March 7, 2020 [9]. Viral spread in Malta was well controlled during the first wave but poorly so during the second wave and to date (March 2021) $[9,10]$. The aim was to explore the impact of COVID-19 through attitudes and experiences on the health and well-being of the Malta adult population suffering from NCDs, a year into COVID-19 pandemic. These data provide an understanding of the impact of COVID-19 on the NCD population's medical care and help us identify whether future targeted action plans are required.

\section{Materials and Methods}

An anonymous survey was designed by the authors targeting the adult population of Malta (a copy found as online suppl. Material; see www.karger.com/doi/10.1159/000519570 for all online suppl. material). At the time the survey was distributed (February 2021), Malta was experiencing a high positivity and mortality rate. However, the vaccination roll-out was progressing quicker than anticipated, with the majority of the healthcare workers, nursing homes, and individuals aged $70+$ years already been vaccinated with 1 dose/fully vaccinated/recieved invitation for vaccination.

Effect of COVID-19 on Non-

Communicable Disease Population
The questionnaire gathered participants' socio-economic data. It assessed for the impact of COVID-19 on their medical care by noting whether the participants visited their general practitioner (GP) less often than before COVID-19 and the reason for this. The participants were asked to note down if they suffered from any of the following NCDs: heart problems, high blood pressure, stroke, type 2 diabetes, type 1 diabetes, respiratory problems, thyroid problems, kidney problems, circulation problems and mental health conditions. Participants were allowed to choose more than one NCDs as well as note down any other concurrent disease/s not mentioned. This study also assessed whether their NCD management was impacted by COVID-19 through postponed or cancelled medical appointment/s or for other reasons. Questions on whether COVID-19 was acquired and the intention to take COVID-19 vaccine were also asked. The survey was distributed through social media (Facebook and LinkedIn) between February 1 and 26, 2021 by using Google Forms ${ }^{\circledR}$ platform.

\section{Data Analyses}

The data gathered were extracted to a spreadsheet and then transferred to statistical software (IBM Corp. Released 2012. IBM SPSS Statistics for Windows, version 21.0; IBM Corp., Armonk, NY, USA) for descriptive analyses. All data gathered in the survey were in a categorical format. Descriptive analyses performed in this study were measures of frequencies through the count function and percentage calculations. These calculations were used to construct figures and tables as well as identify any differences between categories. Categorical comparisons hypothesis was tested statistically by using the $\chi^{2}$ test. A $p$ value of $<0.05$ was considered as statistically significant. For the prevalence results, the corresponding 95\% confidence intervals were also worked out.

Qualitative analysis was performed on participants' comments on their medical care experience/s during the COVID-19 pandemic. Common themes were identified and reported. Ethical clearance was obtained from the University of Malta Research Ethics Committee (ID: 7712_31012021). Participants provided consent when opting to participate in the survey.

\section{Results}

A total of 1,034 participants responded to the online survey, out of which $34.82 \%$ (95\% CI, 31.97-37.77; $n=$ $360)$ reported to suffer from NCDs. The occurrence of a single NCD was reported by $26.69 \%$ (95\% CI, $24.08-$ $29.47 ; n=276), 2$ concurrent diseases by $5.42 \%$ (95\% CI, $4.18-6.98 ; n=56)$, and 3 concurrent diseases by $2.71 \%$ (95\% CI, $1.86-3.90 ; n=28)$. Hypertension $(n=69 ; 6.67 \%)$ and mental disorders $(n=60 ; 5.80 \%)$ were the commonest occurring single NCDs. The combination of hypertension and mental disorders $(n=10 ; 1 \%)$, heart problems and mental disorders $(n=10 ; 1 \%)$, and hypertension and type 2 diabetes $(n=10 ; 1 \%)$ were the commonest concurrent NCDs. Some participants also reported that they suffered additional NCDs than those noted in the survey. The most common NCDs were fi- 
Table 1. Distribution of the sociodemographic characteristics across the participants with reported chronic diseases

\begin{tabular}{|c|c|c|c|c|}
\hline Sociodemographic variables & $\begin{array}{l}\text { Single disease } \\
(n=276), \%\end{array}$ & $\begin{array}{l}\text { Two NCDs } \\
(n=56), \%\end{array}$ & $\begin{array}{l}\text { Three NCDs } \\
(n=28), \%\end{array}$ & $p$ value \\
\hline \multicolumn{5}{|l|}{ Gender } \\
\hline Male & 25.72 & 21.43 & 35.71 & \multirow{2}{*}{0.1} \\
\hline Female & 74.28 & 78.57 & 60.71 & \\
\hline \multicolumn{5}{|l|}{ Age groups, years } \\
\hline $18-19$ & 3.62 & 3.57 & 0.00 & \multirow{8}{*}{$<0.001$} \\
\hline $20-29$ & 13.04 & 7.14 & 0.00 & \\
\hline $30-39$ & 19.93 & 19.64 & 14.29 & \\
\hline $40-49$ & 23.19 & 16.07 & 21.43 & \\
\hline $50-59$ & 21.38 & 26.79 & 28.57 & \\
\hline $60-69$ & 12.68 & 14.29 & 17.86 & \\
\hline $70-79$ & 5.80 & 12.50 & 17.86 & \\
\hline 80 and above & 0.36 & 0.00 & 0.00 & \\
\hline \multicolumn{5}{|l|}{ Education level } \\
\hline Up to secondary school & 16.67 & 10.71 & 25.00 & \multirow{4}{*}{0.01} \\
\hline Up to sixth form & 15.58 & 3.57 & 14.29 & \\
\hline Undergraduate degree & 34.42 & 42.86 & 25.00 & \\
\hline Postgraduate degree & 33.33 & 42.86 & 35.71 & \\
\hline \multicolumn{5}{|l|}{ Employment status } \\
\hline Employed & 67.75 & 64.29 & 60.71 & \multirow{5}{*}{$<0.001$} \\
\hline Unemployed & 0.72 & 3.57 & 3.57 & \\
\hline Student & 9.06 & 5.36 & 0.00 & \\
\hline Retired & 14.49 & 19.64 & 32.14 & \\
\hline Stay at home & 7.97 & 7.14 & 3.57 & \\
\hline
\end{tabular}

NCDs, non-communicable diseases.

bromyalgia $(n=5 ; 0.48 \%)$, neurological problems $(n=4$; $0.39 \%)$, high cholesterol $(n=8 ; 0.77 \%)$, and polycystic ovarian syndrome $(n=4 ; 0.39 \%)$. Online supplementary Table 1 presents the different NCD combinations that were reported by the participants, while Table 1 illustrates the socio-economic characteristics of the participants with NCDs. Of note, the occurrence of NCDs was reported from a young age (18-19 years), which itself is of public health concern.

\section{Impact of Medical Care during COVID-19}

A reduction in GP visits was reported by participants suffering from NCDs (47\%) compared to those without NCDs (32\%) ( $p \leq 0.001)$. A number of reasons were identified for this reduction, as shown in Figure 1. In general, a reduction in sick days was reported across the participants, coinciding with social distancing and hand hygiene advocacy as well as the shifting to remote work by most of the participants. However, concern and fear of acquiring the viral infection if visiting the doctor or leaving the house was significantly observed, especially among those with NCDs $(p \leq 0.001)$. This was further expressed by participants reporting "I rather skip check-ups than risk getting COVID-19 in waiting room or clinic"; "I have skipped routing tests to avoid any contacts. I have only visited the dentist as I had no other options"; and "I am still waiting for the vaccine to go to my dentist and ophthalmologist."

It was observed that COVID-19 had a negative impact on the healthcare system and consequentially on the medical care especially among those with NCDs, as shown in Figure 2. Postponement of appointments was mostly observed for those suffering from 3 concurrent NCDs when comparing with those without any NCDs (single NCD 17\%; 2 NCDs 34\%; 3 NCDs $50 \%$; No NCDs $7 \%$; $p \leq$ $0.01)$. Indeed, it was reported, "delayed treatment and still awaiting further appointments that were postponed more than once"; "awaiting operation, due to COVID-19 several operations were cancelled and thus I need to wait for a longer time"; and "I usually visit my oncologist every 6 months. I already missed 2 visits." This led to a number of participants resorting to private paying services for consultations "I had to go privately" and for blood testing "had to do blood tests privately as health centre was not 


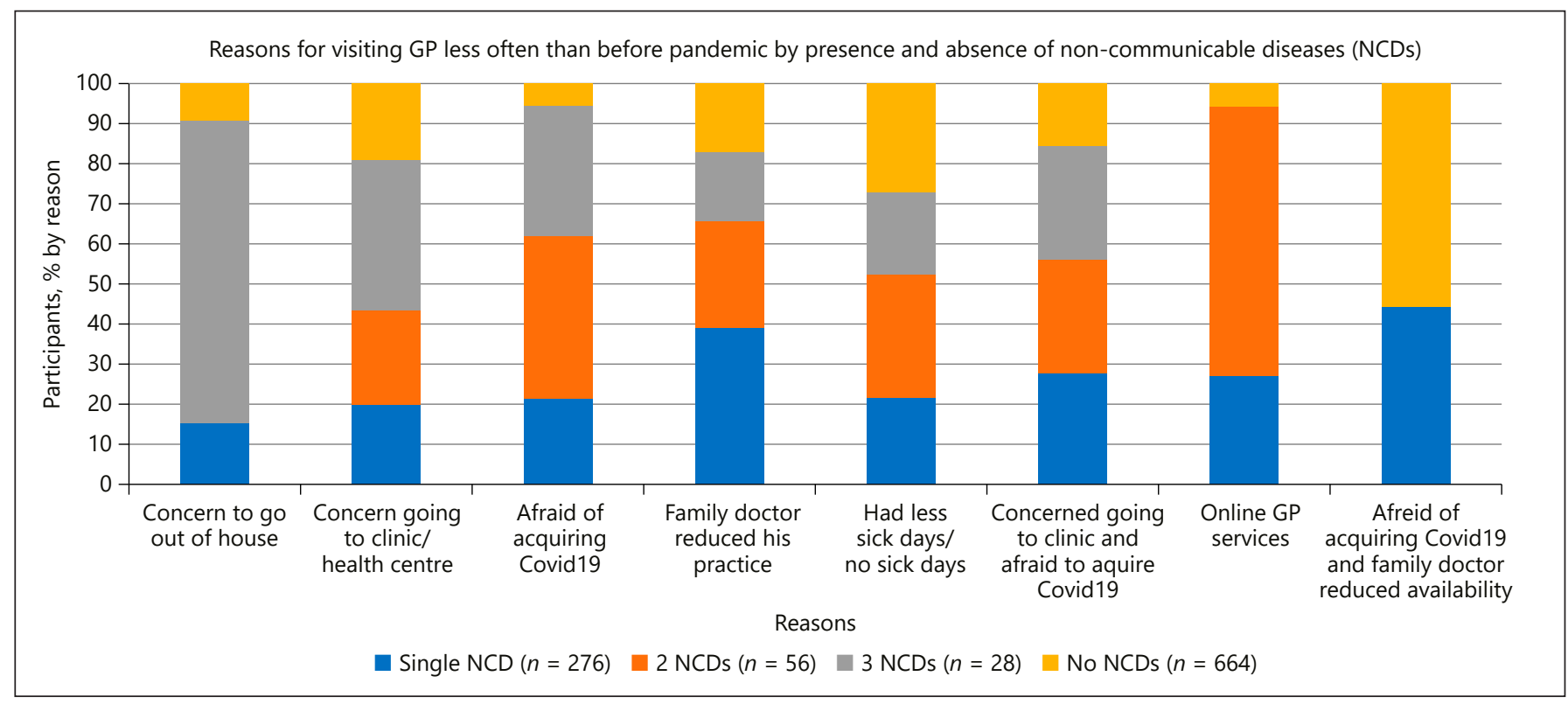

Fig. 1. Different reasons as to the reduction in GP consultation during COVID-19 by the study's participants (staked bar charts). GP, general practitioner; NCDs, non-communicable diseases.

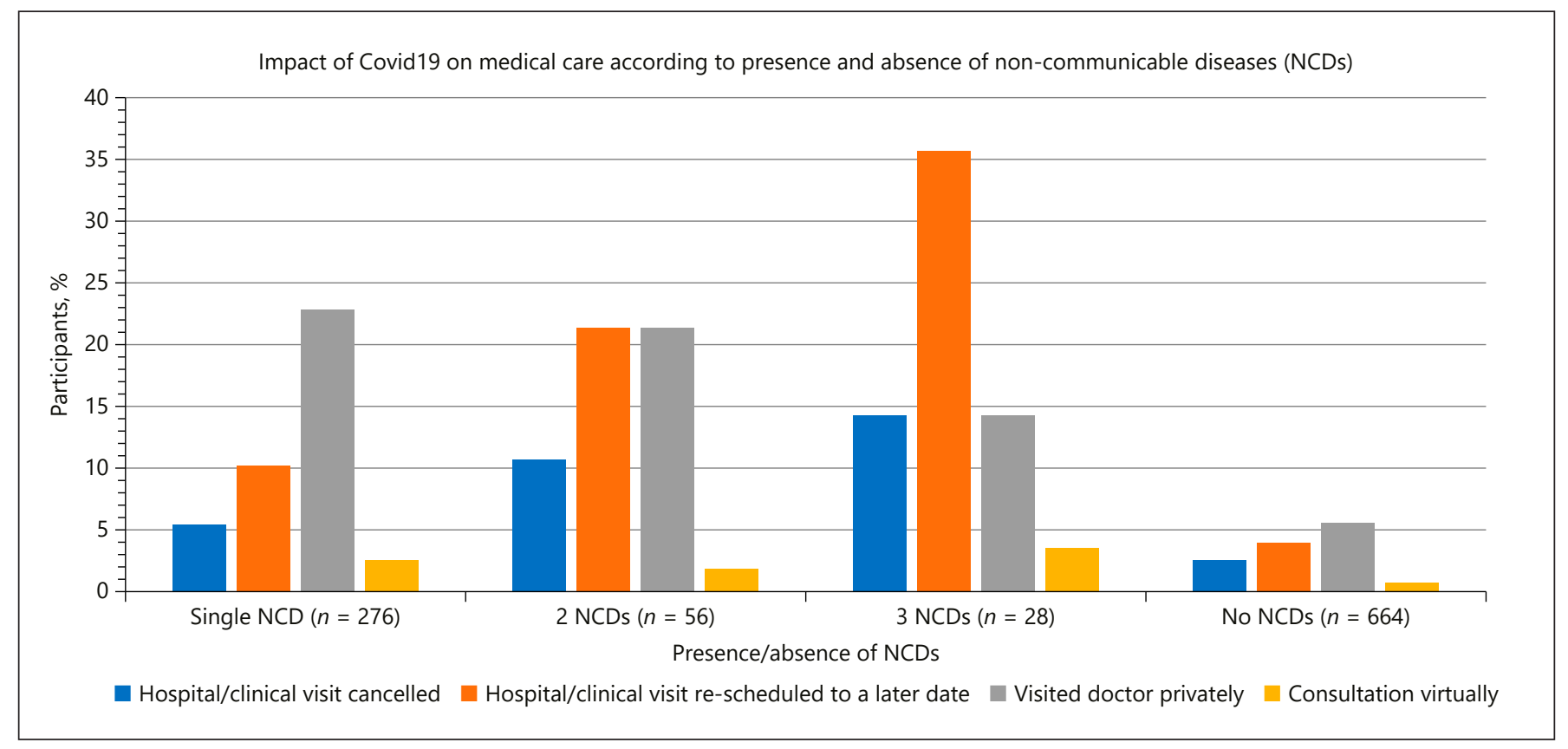

Fig. 2. Impact of COVID-19 on medical care among the study participants (bar graphs). NCDs, non-communicable diseases.

doing them." Of note, a proportion of the participants suffering from NCDs acquired the viral infection of COVID-19 $(6.52 \%$ of the single diseases; $8.93 \%$ of the 2 NCDs; $7.14 \%$ of the 3 NCDs).

Effect of COVID-19 on Non-

Communicable Disease Population
Intention to Take COVID-19 Vaccine

The majority of the participants reported an intention to take the COVID-19 vaccine $(75.39 \%$; 95\% CI, $72.66-$ 77.93), while a proportion had taken the vaccine already 
Fig. 3. Intension to take COVID-19 vaccine among the study's population according to the presence or absence of NCDs (staked bar charts). NCD, non-communicable disease.

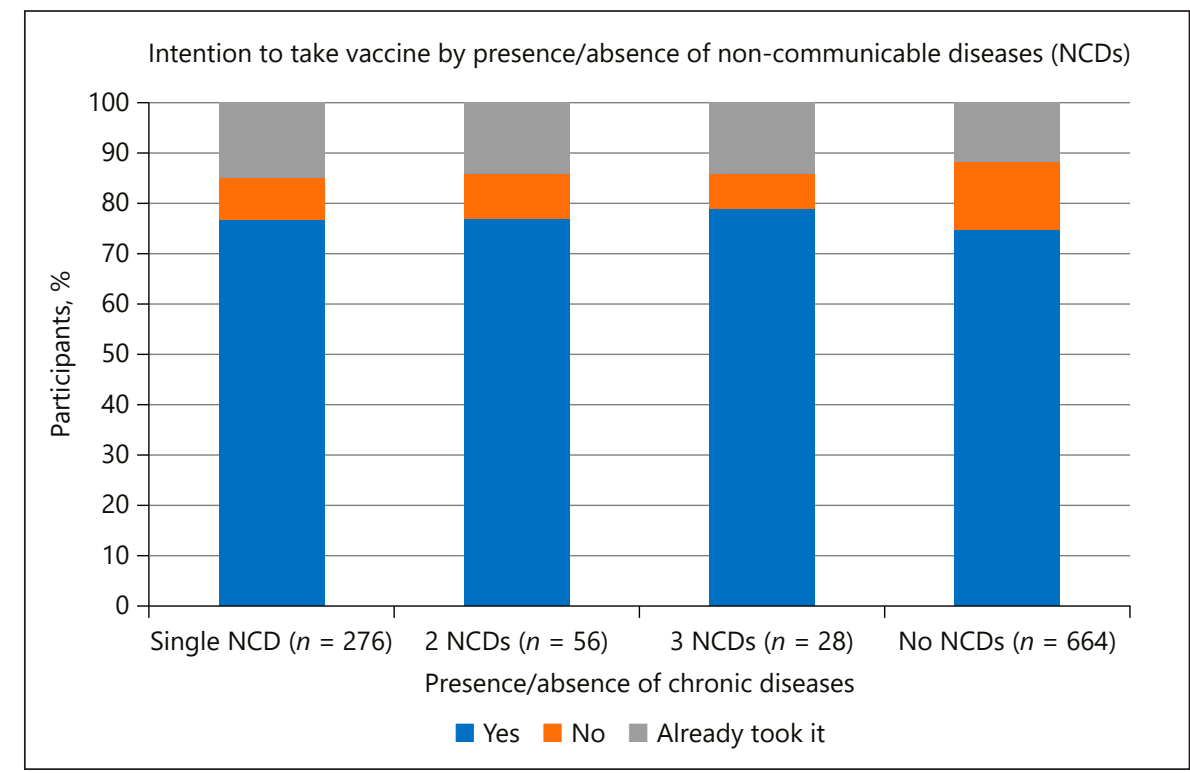

(12.99\%; 95\% CI, 11.06-15.19), as shown in Figure 3. Concerns about the vaccine's safety and long-term side effects were the primary reasons for the small proportion of the individuals that showed reluctancy to take the vaccine.

\section{Discussion}

It has been a year since COVID-19 pandemic took the world hostage, exponentially burdening individuals, healthcare systems, and countries. Like the rest of the world, a number of restrictive measures were instituted as COVID-19 spread through the Maltese islands, including the cancellation and postponement of healthcare appointments $[9,11]$. This coincides with the experiences shared by our study's participants. Of concern is the fact that participants reported that they were still pending appointments (in February 2021), considering that healthcare services were gradually made operational during late Spring and early Summer of 2020 [12]. Resorting to private clinics for consultations or blood testing is a concern since this is a clear example of health inequalities. While the privileged individuals within the upper social gradient can afford such healthcare services, the less advantaged population within the lower social gradient may not. It is known that NCDs are more likely to be prevalent within the low socio-economic groups [13]. Hence, the presence of health inequalities is anticipated to result in disease progression and development of complications within the less privileged population. Inevitably this con- tributes to an increased burden on the population's health, emergency healthcare system, and the economy. Besides, the population suffering from NCDs have been reported to be more susceptible to COVID-19 complications should they become infected [14]. In fact, COVID-19 admission rates to hospital and intensive care units were reported to be predominant among those suffering from chronic diseases, even within Malta [15-17].

This study observed that individuals did not feel safe and expressed concern at the prospect of leaving their homes to seek medical care or attend medical consultations. Such personal decisions are expected to have a negative repercussion on the short- and long-term treatment of NCDs. It is worth noting that during this study's time period (February 1 till 26, 2021), Malta was experiencing a high daily positive cases (average daily cases of 31 per 100,000 ) and deaths (total of 46 deaths) [18]. It is anticipated that such limited healthcare access will also have a negative impact on screening for newly diagnosed or atrisk populations. This will increase the incidence of NCDs and propensity for complications.

Both hypertension and mental health disorders were common occurrences among this study NCD population. Although recall bias cannot be excluded, the fact that the participants noted the presence of such medical problems merits attention. Indeed, a pre-COVID-19 national study conducted among Maltese adults has already reported a substantial prevalence for both diseases (hypertension $30.12 \%$; depression $17.15 \%)[19,20]$. Hence, disruptions of healthcare services along with self-isolation measures 
are expected to have aggravated such problems. Furthermore, it has been reported that since the onset of COVID-19, the burden of mental health among populations has heightened [21]. Therefore, it is envisaged that mental health problems among the population are worse than previously recorded and merit urgent action.

On a positive note, it was clear that the study's participants expressed a definite intention to take the COVID-19 vaccine, within minimal vaccine hesitancy. This attitude illustrates that the general adult population has accepted the COVID-19 vaccine and has understood its importance. Indeed, the COVID-19 vaccine has been labelled as the "magic bullet" against the pandemic [22].

\section{Implications for Policy and Practice}

Focussing on just COVID-19 is set to cause major negative repercussions. Hence, a dual action plan is required to target both COVID-19 and NCDs [16]. It is acknowledged that COVID-19 has added an additional burden to the population and healthcare services. However, NCDs have been declared a global epidemic long before the onset of this pandemic. It is essential that public health authorities along with healthcare services embrace a dual action plan also known as a syndemic approach. Such an approach will ensure that both epidemics (COVID-19 and NCDs) are managed in an adequate manner and prevent complications. This should also include psychological and social assessments. Preventive actions should be set up, including screening for the development of new onset NCDs and/or long COVID-19 syndrome among those having acquired COVID-19 [16,23]. Such screening strategies should be available from a young age, since as observed in this study, occurrence of NCDs is evident from young adulthood. One needs to appreciate that not every country/region/state may have the infrastructure or human resources to set up such an approach. Nonetheless, it is essential that NCDs are brought back to the health agen$\mathrm{da}$ and targeted with the resources that are available.

\section{Study Strengths and Limitations}

The distribution of the survey through social media appeared to have been well accepted by the adult population, managing to capture a good proportion of adults across different socio-economic strata. However, a low response was achieved across the older age groups $(60+$ years), possibly due to lack of social media accounts or information technology illiteracy. Nonetheless, considering that public health preventive interventions should target the young adult generation to enhance the quality of life and decrease long-term complications, this study provides a satisfactory insight on impact of NCDs during COVID-19. This was a self-reported survey and susceptible to self-reporting and recall bias. Furthermore, since the survey was available on social media and anonymous data were gathered, anyone from across the globe could have accessed the survey and participated.

\section{Conclusions}

The COVID-19 pandemic is here to stay for the foreseeable future, but the epidemic of NCDs has been with us for decades. It is clear that individuals with NCDs have experienced a general negative effect on their health care, while embracing vaccination. It is recommended that a dual action strategy is embraced to ensure that both NCDs and COVID-19 are treated simultaneously.

\section{Acknowledgements}

No acknowledgement to declare.

\section{Statement of Ethics}

The study was conducted following the World Medical Association Declaration of Helsinki ethical guidelines. This study protocol was reviewed and approved by the University of Malta Research Ethics Committee (ID: 7712_31012021). Participants provided written informed consent to participate when opting to participate in the survey.

\section{Conflict of Interest Statement}

The authors have no conflicts of interest to declare.

\section{Funding Sources}

No funding was received.

\section{Author Contributions}

S.C. designed the study, S.C. and S.G. analysed the data, S.C. wrote the draft article, S.G. and V.G. reviewed the article, and V.G. supervised the study. All authors have read and approved the manuscript.

\section{Data Availability Statement}

The questionnaire used in this study has been provided as part of the online supplementary material. Data are available upon request. 


\section{References}

1 GBD 2019 Diseases and Injuries Collaborators. Global burden of 369 diseases and injuries in 204 countries and territories, 19902019: a systematic analysis for the Global Burden of Disease Study 2019. Lancet. 2020;396: 1204-22.

2 Maani N, Abdalla SM, Galea S. Avoiding a legacy of unequal non-communicable disease burden after the COVID-19 pandemic. Lancet Diabetes Endocrinol. 2021;9(3):133-5.

3 Shamasunder S, Holmes SM, Goronga T, Carrasco H, Katz E, Frankfurter R, et al. COVID-19 reveals weak health systems by design: why we must re-make global health in this historic moment. Glob Public Health. 2020;15:1083-9.

4 World Health Organization (WHO). Transmission of SARS-CoV-2: implications for infection prevention precautions. World Health Organization, 2020.

5 Hoffmann M, Kleine-Weber H, Schroeder S, Krüger N, Herrler T, Erichsen S, et al. SARSCoV-2 cell entry depends on ACE2 and TMPRSS2 and is blocked by a clinically proven protease inhibitor. Cell. 2020;181(2):271-80. e8.

6 Simões e Silva AC, Silveira KD, Ferreira AJ, Teixeira MM. ACE2, angiotensin-(1-7) and Mas receptor axis in inflammation and fibrosis. Br J Pharmacol. 2013;169:477-92.

7 Bornstein SR, Dalan R, Hopkins D, Mingrone G, Boehm BO. Endocrine and metabolic link to coronavirus infection. Nat Rev Endocrinol. 2020;16:297-8.

8 Kluge HHP, Wickramasinghe K, Rippin HL, Mendes R, Peters DH, Kontsevaya A, et al. Prevention and control of non-communica- ble diseases in the COVID-19 response. Lancet. 2020;395(10238):1678-80.

9 Cuschieri S. COVID-19 panic, solidarity and equity-the Malta exemplary experience. Z Gesundh Wiss. 2020:1-6. Epub ahead of print.

10 Cuschieri S, Balzan M, Gauci C, Aguis S, Grech V. Mass events trigger malta's second peak after initial successful pandemic suppression. J Community Health. 2020:1-8. Epub ahead of print.

11 Chudasama YV, Gillies CL, Zaccardi F, Coles B, Davies MJ, Seidu S, et al. Impact of COVID-19 on routine care for chronic diseases: a global survey of views from healthcare professionals. Diabetes Metab Syndr. 2020;14: 965-7.

12 Cuschieri S. COVID-19: the transition towards a new normal - experiences from the European country of Malta. J Public Health. 2021:1-8. Epub ahead of print.

13 Oates GR, Jackson BE, Partridge EE, Singh KP, Fouad MN, Bae S. Sociodemographic patterns of chronic disease: how the mid-South region compares to the rest of the country. Am J Prev Med. 2017;52:S31-9.

14 The Lancet. COVID-19: a new lens for noncommunicable diseases. Lancet. 2020;396: 649.

15 O'Donovan MR, Sezgin D, Liew A, O'Caoimh R. Burden of disease, disability-adjusted life years and frailty prevalence. QJM. 2019;112: 261-7.

16 Azarpazhooh MR, Morovatdar N, Avan A, Phan TG, Divani AA, Yassi N, et al. COVID-19 pandemic and burden of non-communicable diseases: an ecological study on data of 185 countries. J Stroke Cerebrovasc Dis. 2020;29:105089.

17 Micallef S, Piscopo TV, Casha R, Borg D, Vella C, Zammit MA, et al. The first wave of COVID-19 in Malta; a national cross-sectional study. PLoS One. 2020;15:e0239389.

18 COVID-19 Public Health Response Team Ministry for Health. COVID-19 data management system. 2021. Available from: https: //deputyprimeminister.gov.mt/en/healthpromotion/covid-19/Pages/covid-19-infographics.aspx.

19 Cuschieri S, Vassallo J, Calleja N, Pace N, Mamo J. The effects of socioeconomic determinants on hypertension in a cardiometabolic at-risk European country. Int J Hypertens. 2017;2017:7107385.

20 Cuschieri S, Mamo J. Are normoglycaemic individuals at risk of depression? The depression-dysglycaemic phenotype from a European population-based cross-sectional study. Arch Public Health. 2020;78:111.

21 Pan KY, Kok AAL, Eikelenboom M, Horsfall M, Jörg F, Luteijn RA, et al. The mental health impact of the COVID-19 pandemic on people with and without depressive, anxiety, or obsessive-compulsive disorders: a longitudinal study of three Dutch case-control cohorts. Lancet Psychiatry. 2021;8(2):121-9.

22 Todd A, Bambra C. Learning from past mistakes? The COVID-19 vaccine and the inverse equity hypothesis. Eur J Public Health. 2021; 31(1):2.

23 Gorna R, MacDermott N, Rayner C, O'Hara M, Evans S, Agyen L, et al. Long COVID guidelines need to reflect lived experience. Lancet. 2021;397(10273):455-7. 